\title{
Did the Angiodysplasia Associated With Heyde's Syndrome Disappear Spontaneously?: A Case Report
}

\section{Rui Li ( $\sim 20193109067 @ s t u . g z u c m . e d u . c n)$}

Guangzhou University of Chinese Medicine

Jiechun Zhang

Guangzhou University of Chinese Medicine

Jiaxi Shi

Guangzhou University of Chinese Medicine

\section{Lijin Qing}

The First Affiliated Hospital of Guangzhou university of Chinese Medicine

\section{Wei Wu}

The First Affiliated Hospital of Guangzhou University of Chinese Medicine

\section{Case report}

Keywords: Heyde's Syndrome, Aortic valve stenosis, Gastrointestinal bleeding, von Willebrand factor, case report

Posted Date: September 20th, 2021

DOI: https://doi.org/10.21203/rs.3.rs-792737/v1

License: (c) (i) This work is licensed under a Creative Commons Attribution 4.0 International License. Read Full License 


\section{Abstract}

Background: Multidisciplinary clinical manifestations of Heyde's syndrome and finite accuracy of corresponding examinations toward Heyde's triad make it easily omitted or misjudged in practice. Moreover, aortic valve replacement is often delayed because of the contradiction between anticoagulation and hemostasis. Herein, we present a rare case of atypical Heyde's syndrome whose confirmed intermittent bleeding angiodysplasia was not observed via mesenteric arteriography again, but severe bleeding was dramatically improved by transcatheter aortic valve implantation(TAVI) following laparotomy.

Case presentation: A 64-year-old female experienced refractory gastrointestinal bleeding and deteriorating exertional dyspnea with a history of hypertension. Exploratory laparotomy was performed because the hemorrhage persisted and repeated transfusions. The subsequent histological examination revealed angiodysplasia. Heyde's syndrome was not suspected until she bled again combined with aortic valve stenosis detected by echocardiography 3 years later. TAVI was consequently performed in a stable condition with the invisibility of angiodysplasia. The post-procedure and follow-up were uneventful.

Conclusions: The visible figures of angiodysplasia or shortage of HMWM-vWFs should not be indispensable for the clinical diagnosis of Heyde's syndrome. Laparotomy could be a bridging therapy to aortic valve replacement for severe hemorrhagic patients, and TAVI may benefit high-risk patients with a stable condition.

\section{Background}

The incidence and mortality of Heyde's syndrome have been on the rise for nearly a decade(1). The most prominent pathomechanism is deemed an acquired deficiency of HMWM-vWFs. Although there are not yet guidelines for Heyde's Syndrome, it is generally believed that its diagnosis should be based on a triad of AS, refractory GIB, and associated angiodysplasia or AVWS(2). However, its multidisciplinary clinical manifestations and finite accuracy of corresponding examinations make it easily omitted or misjudged. Moreover, aortic valve replacement is often delayed because of the contradiction between anticoagulation and hemostasis(3). Herein, we summarized a case of Heyde's syndrome that angiodysplasia was not detected again before operation but was absolutely benefited from TAVI following laparotomy.

\section{Case Presentation}

A 64-year-old woman usually experienced chest distress and shortness of breath during moderate activity. She had no medical history but ten years' hypertension.

In February 2014, she suffered repetitive episodes of tarry stool accompanied by palpitations, chest tightness, and dizziness without any apparent incentives. Gastroduodenoscopy and colonoscopy did not 
locate the lesions of bleeding. Subsequent capsule enteroscopy identified multiple hemorrhagic angiodysplasia besides the diverticulum in the jejunum.

Nevertheless, there is little hemostatic effect following endoscopic argon plasma coagulation and conventional medication. She had to receive blood transfusion for several units, during which her $\mathrm{Hb}$ fluctuated between 74 and 124 (normal 110-150g/L). A few months later, as hemorrhage persisted and repeated transfusions were necessary, an exploratory laparotomy was performed, and the suspicious lesion in the intestine was resected. The relevant histological examination showed chronic mucosal inflammation with multiple erosions compatible with intestinal angiodysplasia. Thus, thalidomide $(25 \mathrm{mg} / \mathrm{d})$ was prescribed for preventing neoangiogenesis postoperatively. Her GIB was consequently ceased for some time.

In May 2017, the patient was sent to receive an emergency coronary angiography because of syncope, and it revealed $40 \%$ diffuse stenosis in the middle right coronary artery with no further critical lesions. She had melena again after the procedural that may relate to the prophylaxis of dual antiplatelet therapy,which was controlled by conventional medications such as PPI and octreotide.

Afterward, the patient was referred to our department due to worsening exertional dyspnea, thoracalgia, and orthostatic hypotension. Her clinical signs of AS and systemic congestion were obvious. Laboratory tests were unremarkable other than $\mathrm{Hb} 76 \mathrm{~g} / \mathrm{L}$, NT-proBNP 392(normal < 125) pg/mL. TTE showed severe calcified AS with mean and peak transvalvular pressure gradient of 48 and $84 \mathrm{mmHg}$, respectively; aortic valve area, $0.80 \mathrm{~cm}^{2}$; without any signs toward obstructive HCM. (Fig. 1A-C)

In August 2018, the patient underwent TAVI with VENUS-A 26 prosthetic aortic valve in a stable condition without any remarkable paravalvular leak. Interestingly, it is difficult to find arteriovenous malformations via superior mesenteric artery angiography before TAVI. The peak aortic valve pressure gradient improved from $88 \mathrm{mmHg}$ to less than $5 \mathrm{mmHg}$ postprocedurally, and she had neither enterorrhagia nor major bleeding during the perioperative period. For her antithrombotic strategy, clopidogrel would be prescribed for 1-year besides Pradaxa for long-term administration.

At 1-year follow-up, the TTE confirmed a well-seated prosthesis with a peak transvalvular pressure gradient of $12 \mathrm{mmHg}$; and aortic valve area, $4.11 \mathrm{~cm}^{2}$; excepted for emerging simple LVOTO which was not accompanied by systolic anterior motion of the mitral valve or HCM. (Fig. 1D-E) Hitherto, she was free from any significant hemorrhagic or ischemic events as well as the triad of AS.

\section{Discussion}

\section{Epidemiology and Pathophysiology}

The prevalence of Heyde's syndrome in patients with severe AS increased annually along with the aggravation of aging society and reached $1.87 \%$ to $3.2 \%$ currently, resulting in escalating mortality of the disorder.(1) The corresponding dominating mechanism is the excessive proteolysis of HMWM-vWFs by 
ADAMTS-13 around the context of high shear stress related to stenotic valves, which not only predisposes to bleed but also induces submucosal arteriovenous malformation in the digestive tract(4, $5)$.

\section{Diagnosis}

It is generally believed that diagnosing Heyde's syndrome should be based on a triad of AS, refractory GIB, and associated angiodysplasia or AVWS. Meanwhile, other diseases that can cause GIB such as tumors, primary digestive, hematological or autoimmune diseases, and drug-induced effects should be ruled out.

As for AS, it is known that patients with severe AS have a 100 times risk of complicating hematochezia than those without(6). Our patient's symptoms of continuous dyspnea, chest pain, palpitations, and even syncope during mild to moderate exercise should be aware of a triad of AS earlier after excluding severe coronary artery disease, so as to differentiate from Heyde's syndrome timely.

As for angiodysplasia, locating the culprit lesion can be challenging. Despite the rapid development of endoscopic diagnostic technologies including capsule endoscopy for a decade, $35 \%$ of cases of angiodysplasia were undiagnosed(3). Although the gold standard diagnostic method is mesenteric arteriography, the mean rate of localization of the bleeding site is $40 \%$ since many cases of bleeding angiodysplasia are intermittent which will decrease its sensitivity on detection(7). Our patient's angiodysplasia was confirmed by histological examination through exploratory laparotomy. Nevertheless, it was not able to be observed via mesentericography several years later. It might be primarily attributed to both the primary suspicious lesion in the gut had been surgically removed or restricted by thalidomide, and the patient was non-bleeding when receiving inspection.

As for AVWS, the necessity of gel electrophoresis to confirm the loss of large multimers makes biologic diagnosis of Heyde's syndrome challenging. The coagulopathy may be absent in patients with aortic gradients below $50 \mathrm{mmHg}$ besides its high cost and time-consuming(2). The vWFs were not examined in our patient due to the limited conditions of our laboratory department. Research shows that the prevalence of abnormality of HMWM-vWFs in patients with native AS is estimated from $65 \%$ to $92 \%$, and bleeding angiodysplasia in patients with AVWS is $11.5 \%$ approximately. Meanwhile, $55.6 \%$ to $87.5 \%$ of patients with documented angiodysplasia have a deficiency of HMWM-vWFs(8). However, other studies showed that patients with bleeding angiodysplasia did not have an increased prevalence of AVWS(9). In the context of AS, other mechanisms such as low perfusion, submucosal ischemia and hypoxia, or cholesterol embolism have been advocated to explain the relationship among angiodysplasia, GIB and AS(10).

We searched for case reports of Heyde's syndrome published since 2000 in PubMed, revealing 91 articles. We excluded 13 articles (1) without obtaining relevant information $(n=10),(2)$ not consistent with the disease $(n=1),(3)$ the case of epistaxis $(n=1)$, and (4) the patient is an infant $(n=1)$. We summarized the remaining 78 articles that a total of 83 cases ( 5 articles were double-case reports) with special references 
to treatment methods, angiodysplasia and HMWM-vWFs (Supplement table 1). Among them, the primary diseases were severe LVOTO in 6 cases, severe aortic regurgitation in 1cases, AS in the remaining 76 cases; 17 cases (20.5\%) were not diagnosed with angiodysplasia, of which, 11 (84.6\%) of 13 patients undergoing cardiac surgery (including aortic valve replacement and alcohol septal ablation) were cured of GIB; 9 cases (10.8\%) had not HMWM-vWFs deficiency, 46 cases $(55.4 \%)$ were not examined for HMWM-vWFs, of which, 31 (91.2\%) of 34 cases receiving cardiac surgery had remission of GIB; 10 patients were not diagnosed with both angiodysplasia and AVWS, of which, 6(85.7\%) of 7 patients performed heart surgery were cured of GIB.

Given the complicated mechanisms and imperfect accuracy or availability of relevant diagnostic methods, we should not simply exclude Heyde's syndrome in practice merely due to lack of evidence of bleeding angiodysplasia or AVWS. It is worth paying attention to considering Heyde's syndrome as an exclusionary diagnosis and the feasibility of aortic valve replacement as a diagnostic therapy. $\mathrm{QFig} . \mathbf{2 \square}$

\section{Treatment}

Endoscopic therapies are often ineffective, whereas exploratory laparotomy could be a bridging therapy to aortic valve replacement for patients with continuous enterorrhagia or major or life-threatening hemorrhage. Even though it remains about $30 \%$ of bleeding recrudescence due to angiodysplasia diffusing throughout the digestive tract(11). Our patient underwent segmental enterectomy as severe bleeding and temporarily alleviated after regular administration of thalidomide that the angiogenesis inhibitor.

It is demonstrated that SAVR or TAVI is a radical therapy for Heyde's syndrome. In comparison, TAVI may be an optimal modality for stable patients at high risks such as agedness, multiple comorbidities, or hemorrhagic predisposition. Desai et al.(1) found no significant differences between TAVI and SAVR in all-cause mortality or total expenses during hospitalization. Moreover, TAVI is superior to SAVR in lessening the duration of hospitalization and the incidence of periprocedural complications such as stroke, myocardial infarction, or major or life-threatening bleeding. Nevertheless, TAVI is pointed out to be prone to complicate paravalvular regurgitation $₫ 26.6 \%$ vs. $4.2 \%, P<0.001$ \and associated with a higher incidence of advanced GIB $₫ 3.3 \%$ vs. $1.5 \%, P<0.001 \rrbracket$ compared to $\operatorname{SAVR}(12,13)$. Our patient, categorized as the population of extremely high bleeding risk in operation, consequently received TAVI to treat to avoid surgical complications and increase the benefit of valve replacement.

Although there are some case reports of Heyde's syndrome toward severe LVOTO, we have not seen any prognostic reports concerning the complication of LVOTO after TAVI in patients with Heyde's syndrome. A study indicated that $89 \%$ of patients with baseline or latent obstructive HCM suffered abnormality of HMWM-vWFs which were enough to be impaired when the peak gradient of the obstructive LVOT is $>15 \mathrm{mmHg}$ (at rest) or $>35 \mathrm{mmHg}$ (during exercise). At the same time, about $30 \%$ of those patients developed $\operatorname{GIB}(14,15)$. Our patient, verified as having no myocardial hypertrophy or LVOTO by echocardiography preprocedurally, was found to complicate with asymptomatic and simple LVOTO (maximum peak gradient in LVOT of $55 \mathrm{mmHg}$ ) at 1-year's follow up. It is worth further 
investigating whether or how the complication of LVOTO following TAVI in patients with Heyde's syndrome impacts their prognosis on GIB.

\section{Conclusion}

Elderly patients suffering from either bleeding angiodysplasia,refractory GIB with unclear causes or poor efficacy from conventional gastroenterological therapies should be examined by echocardiography and differentiated from Heyde's syndrome. We should focus on its two main symptoms when diagnosing the syndrome: severe AS and refractory GIB. It should not be simply ruled out just based on the absence of evidence of angiodysplasia or AVWS, but other disorders leading to GIB should be excluded before consideration. Laparotomy could be a bridging therapy to aortic valve replacement for severe hemorrhagic patients, and TAVI may benefit high-risk patients in stable condition.

\section{Abbreviations}

AS aortic valve stenosis

GIB gastrointestinal bleeding

HMWM-vWFs high-molecular-weight multimers of vWFs

AVWS acquired von Willebrand syndrome

LVOTO left ventricular outflow tract obstruction

TAVI transcatheter aortic valve implantation

$\mathrm{Hb}$ hemoglobin concentration

TTE Transthoracic echocardiography

HCM hypertrophic cardiomyopathy

SAVR surgical aortic valve replacement

LVOT left ventricular outflow tract

\section{Declarations}

\section{Acknowledgements}

We appreciate the patient for understanding and cooperation. We thank Dr. Jianfang Luo and his team from Guangdong Provincial People's Hospital for their successful interventional procedures.

\section{Authors' contributions}


The authors' responsibilities were as follows: Wu Wei and Rui Li contributed to the conception or design of the case as well as to the acquisition, analysis, or interpretation of the patient's manifestations; Rui Li drafted the manuscript; Lijin Qing assisted with the treatment of the patient; Jiechun Zhang and Jiaxi Shi critically revised the manuscript. All authors gave their final approval and agreed to be accountable for all aspects of work to ensure the integrity and accuracy of the case.

\section{Funding}

The authors received no financial support for the research, authorship, and/or publication of this article.

\section{Availability of data and materials}

The datasets of the current study are available from the corresponding author upon reasonable request.

\section{Ethics approval and consent to participate}

This study was conducted with approval from the Ethics Committee of The

First Affiliated Hospital of Guangzhou University of Chinese Medicine.

\section{Consent for publication}

Consent for publication is obtained from the patient.

\section{Competing interests}

The authors declare no potential conflicts of interest with respect to the research, authorship, and/or publication of this article.

\section{Author details}

a Guangzhou University of Chinese Medicine, Guangzhou, China

${ }^{b}$ Department of Cardiovascular Medicine, The First Affiliated Hospital of Guangzhou University of Chinese Medicine, No. 16, Ji Chang Road, Baiyun, 510405, Guangzhou, China

\section{References}

1. Desai R, Parekh T, Singh S, Patel U, Fong HK, Zalavadia D, et al. Alarming Increasing Trends in Hospitalizations and Mortality With Heyde's Syndrome: A Nationwide Inpatient Perspective (2007 to 2014). Am J Cardiol. 2019;123(7):1149-55.

2. Blackshear JL. Heyde Syndrome: Aortic Stenosis and Beyond. Clin Geriatr Med. 2019;35(3):369-79.

3. Mohee K, Aldalati O, Dworakowski R, Haboubi H. Aortic stenosis and anemia with an update on approaches to managing angiodysplasia in 2018. Cardiol J. 2020;27(1):72-7. 
4. Okhota S, Melnikov I, Avtaeva Y, Kozlov S, Gabbasov Z. Shear Stress-Induced Activation of von Willebrand Factor and Cardiovascular Pathology. Int J Mol Sci. 2020;21(20).

5. Randi AM, Smith KE, Castaman G. von Willebrand factor regulation of blood vessel formation. Blood. 2018;132(2):132-40.

6. Jehangir A, Pathak R, Ukaigwe A, Donato AA. Association of aortic valve disease with intestinal angioectasia: data from the Nationwide Inpatient Sample. Eur J Gastroenterol Hepatol. 2018;30(4):438-41.

7. Shah AR, Jala V, Arshad H, Bilal M. Evaluation and management of lower gastrointestinal bleeding. Dis Mon. 2018;64(7):321-32.

8. Makris M, Federici AB, Mannucci PM, Bolton-Maggs PH, Yee TT, Abshire T, et al. The natural history of occult or angiodysplastic gastrointestinal bleeding in von Willebrand disease. Haemophilia. 2015;21(3):338-42.

9. Höög CM, Broström O, Lindahl TL, Hillarp A, Lärfars G, Sjöqvist U. Bleeding from gastrointestinal angioectasias is not related to bleeding disorders - a case control study. BMC Gastroenterol. 2010;10:113.

10. Brandão D, Costa C, Canedo A, Vaz G, Pignatelli D. Endogenous vascular endothelial growth factor and angiopoietin-2 expression in critical limb ischemia. Int Angiol. 2011;30(1):25-34.

11. Garcia-Compean D, Del Cueto-Aguilera AN, Jimenez-Rodriguez AR, Gonzalez-Gonzalez JA, Maldonado-Garza HJ. Diagnostic and therapeutic challenges of gastrointestinal angiodysplasias: A critical review and view points. World J Gastroenterol. 2019;25(21):2549-64.

12. Leon MB, Smith CR, Mack MJ, Makkar RR, Svensson LG, Kodali SK, et al. Transcatheter or Surgical Aortic-Valve Replacement in Intermediate-Risk Patients. N Engl J Med. 2016;374(17):1609-20.

13. lyengar A, Sanaiha Y, Aguayo E, Seo YJ, Dobaria V, Toppen W, et al. Comparison of Frequency of Late Gastrointestinal Bleeding With Transcatheter Versus Surgical Aortic Valve Replacement. Am J Cardiol. 2018;122(10):1727-31.

14. Le Tourneau T, Susen S, Caron C, Millaire A, Maréchaux S, Polge AS, et al. Functional impairment of von Willebrand factor in hypertrophic cardiomyopathy: relation to rest and exercise obstruction. Circulation. 2008;118(15):1550-7.

15. Blackshear JL, Stark ME, Agnew RC, Moussa ID, Safford RE, Shapiro BP, et al. Remission of recurrent gastrointestinal bleeding after septal reduction therapy in patients with hypertrophic obstructive cardiomyopathy-associated acquired von Willebrand syndrome. Journal of thrombosis haemostasis: JTH. 2015;13(2):191-6.

\section{Figures}




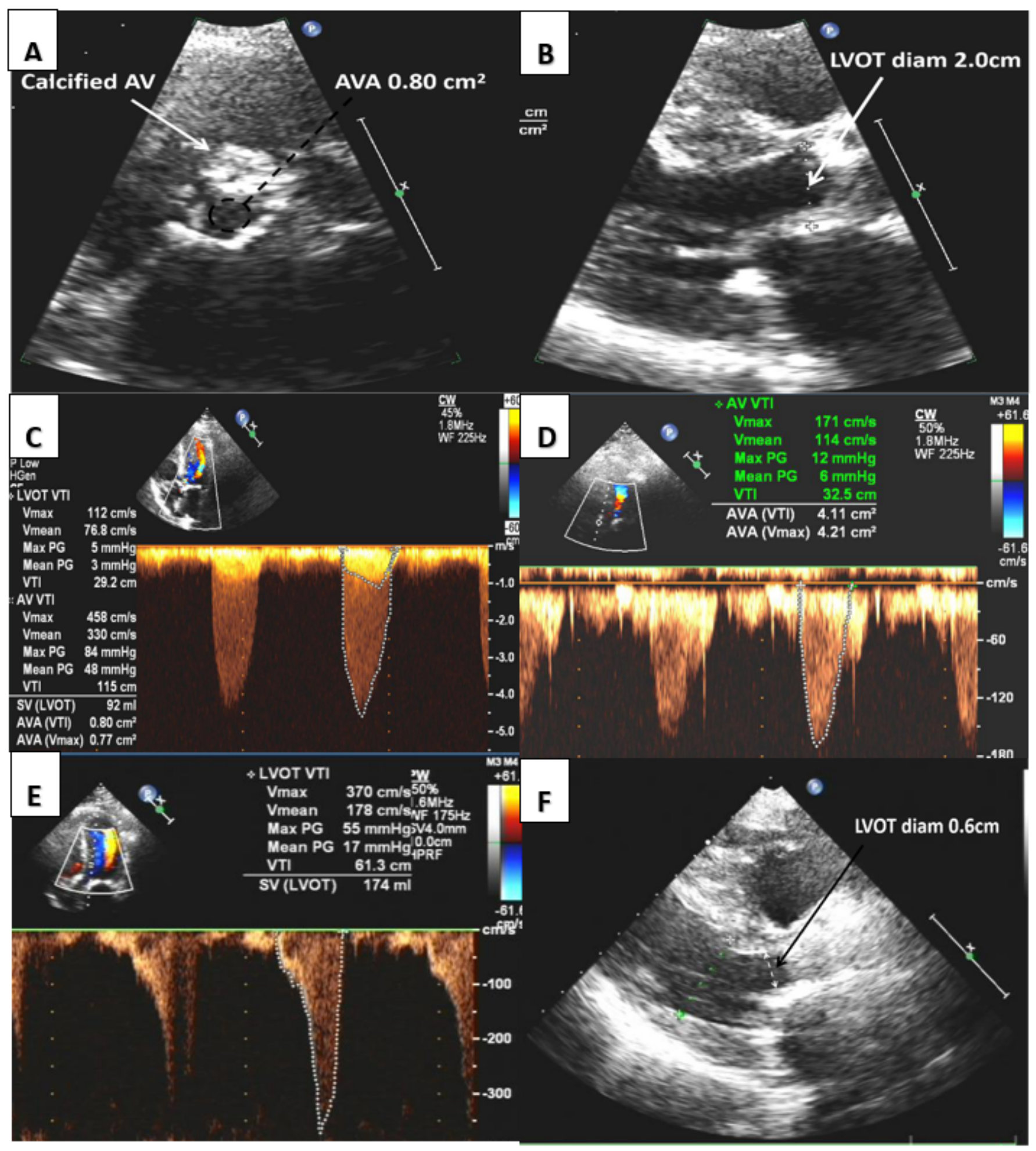

\section{Figure 1}

Pre-procedural TTE shows $(A)$ severe aortic stenosis with calcified aortic valve $(A V)$ and aortic valve area (AVA) of $0.80 \mathrm{~cm} 2$;(B)left ventricular outflow tract(LVOT) diam of $2.0 \mathrm{~cm}$; and(C)maximum peak gradient in LVOT and AV of $5 \mathrm{mmHg}$ and $84 \mathrm{mmHg}$, respectively; maximum systolic flow velocity in LVOT and AV of $1.12 \mathrm{~m} / \mathrm{s}$ and $4.58 \mathrm{~m} / \mathrm{s}$, respectively. TTE at a year follow up shows (D, E) maximum peak gradient in 
LVOT and AV of $55 \mathrm{mmHg}$ and $12 \mathrm{mmHg}$, respectively; maximum systolic flow velocity in LVOT and AV of $3.70 \mathrm{~m} / \mathrm{s}$ and $1.71 \mathrm{~m} / \mathrm{s}$, respectively; AVA of $4.11 \mathrm{~cm} 2$; and $(\mathrm{F})$ LVOT diam of $0.60 \mathrm{~cm}$.

\section{Image not available with this version}

\section{Figure 2}

Flowchart of the diagnosis and treatment of Heyde's Syndrome. GIB: gastrointestinal bleeding; AS: aortic valve stenosis.

\section{Supplementary Files}

This is a list of supplementary files associated with this preprint. Click to download.

- CAREchecklistEnglish2013.pdf

- Supplementtable1jcs.docx 